\title{
Desenvolvimento de um Método para a Determinação da lodúria e sua Aplicação na Excreção Urinária de lodo em Escolares Brasileiros
}

\begin{abstract}
RESUMO
Desenvolvemos método semi-automatizado em placa para a determinação de iodo urinário; utilizamos, primeiramente, a digestão das amostras de urina com persulfato de amônio e, a seguir, estimamos a quantidade de iodo pela redução do sulfato cérico amoniacal. O método foi validado no inquérito nacional de monitoração da deficiência de iodo, realizado em 1994, que empregou um sistema de amostragem mista da população brasileira e analisou a iodúria em 16.803 amostras de urina de escolares obtidas por coleta casual. Em 401 municípios estudados encontramos 4 com deficiência de iodo de grau moderado (Almas, Arraias e Paraná, em Tocantins, e Cocos, na Bahia) e 116 de grau leve. Desta forma, este estudo mostrou a presença de regiões com deficiência de iodo em 1994, a despeito do programa de iodação do sal. Dados recentes de outros autores, com número menor de municípios, indicam excesso de ingestão de iodo. Assim, num país de dimensões continentais e heterogêneo como o Brasil, é necessária a realização de avaliações periódicas de amplitude nacional para a verificação da ingestão de iodo da população. $O$ método apresentado neste estudo apresenta as características de simplicidade e eficiência necessários para este tipo de estudo populacional. (Arq Bras Endocrinol Metab 2007;51/9:1477-1484)
\end{abstract}

Descritores: lodúria; Deficiência de iodo; Bócio endêmico

\section{ABSTRACT}

\section{Development of a Semi-Automated Method for Measuring Urinary lodine and Its Application in Epidemiological Studies in Brazilian Schoolchildren.}

In this study we developed a semi-automated method for the measurement of urinary iodine using firstly ammonium persulfate for digestion of urine followed by estimation of iodine content in the Sandell-Kolthoff reaction, in which iodine acts as a catalyst for the reduction of cerium. This method was validated in the 3rd Brazilian National Survey of iodine deficiency in 1994. We studied 16,803 casual urine samples from schoolchildren of 401 cities and found 4 moderately-deficient towns (Almas, Arraias, and Parana, in the State of Tocantins, and Cocos, in the State of Bahia), and 116 mildly-deficient. This work suggests that despite the salt iodization program, there was some iodine-deficient areas in Brazil in 1994. Recent surveys, involving less cities, are indicating an excess of iodine ingestion. Therefore, in a country of continental dimensions and very heterogeneous in terms of public health, periodical evaluations are necessary to monitor the real situation of iodine nutrition in Brazil. The method developed in this paper is suitable for these surveys. (Arq Bras Endocrinol Metab 2007;51/9:1477-1484)

Keywords: Urinary iodine; lodine deficiency; Endemic goiter artigo original

\author{
ROBERTO Z. ESTEVES \\ TERESA S. KASAMATSU \\ ILDA S. KUNII \\ Gilberto K. FuRUZAWA \\ José Gilberto H. VieIRA \\ RUI M.B. MACIEL
}

Departamento de Medicina,

Centro de Ciências da Saúde,

Universidade Estadual de

Maringá (RZE), PR, e Disciplina

de Endocrinologia,

Departamento de Medicina,

Escola Paulista de Medicina,

Universidade Federal de

São Paulo (TSK, ISK, GKF, JGHV

\& RMBM), São Paulo, SP. 
A DEFICIÊNCIA EM IODO é a principal causa evitável de dano cerebral em fetos e crianças e de retardo do desenvolvimento psicomotor. Trata-se de problema de saúde pública de escala global, envolvendo 130 países; hoje, cerca de 2,2 bilhões de pessoas vivem em regiões carentes em iodo (1-3). O iodo é um microelemento esssencial e sua principal função no organismo é a síntese dos hormônios tiroidianos tiroxina (T4) e triiodotironina (T3); as necessidades dietéticas diárias são entre 100-150 $\mathrm{\mu g} /$ dia para adultos e adolescentes, 60-100 $\mu \mathrm{g} /$ dia para crianças de 1 a 10 anos e $35-40 \mu \mathrm{g} / \mathrm{dia}$ para aquelas com menos de 1 ano (1-3). Se esses níveis não forem atingidos numa determinada região, um amplo espectro de alterações funcionais pode ocorrer. Apesar de essas manifestações serem evidentes em regiões de carência importante de iodo, quando o bócio e o cretinismo são manifestações extremas, nas regiões onde a deficiência for leve ou moderada a endemia tende a ser sub-avaliada, pois ocorrem alterações mais sutis, como hipotiroidismo subclínico, baixo rendimento escolar, aumento da mortalidade perinatal e infantil e estagnação socioeconômica (2-6).

No Brasil, a deficiência de iodo é reconhecida há muito tempo, e desde meados do século passado determinou-se que deveria haver suplementação de iodo por meio da adição deste elemento ao sal de cozinha, de acordo com recomendações da Organização Mundial da Saúde (OMS) (3).

Para avaliar se a população está recebendo o iodo por meio do sal, os inquéritos nutricionais empregam índices indiretos, dos quais os mais utilizados são a prevalência de bócio, examinada pelo exame físico ou por ultra-sonografia cervical e a determinação da excreção urinária de iodo $(1,7)$. Os levantamentos de prevalência de bócio baseiam-se, geralmente, na inspeção e palpação da tiróide. Apesar de a ultra-sonografia permitir determinação mais precisa do volume tiroidiano, é dispendiosa e exige qualificação do examinador, o que limita seu emprego $(1,7,8)$. Para que o bócio possa ser dito endêmico numa determinada região, sua prevalência deve ser maior ou igual a $10 \%$; no entanto, a presença de outras causas ambientais bociogênicas, como o tiocianato e a carência em selênio, fazem com que tal índice nem sempre reflita a situação real da carência em iodo (8).

A concentração de iodo na urina (a excreção renal corresponde a mais de $90 \%$ das perdas e é equivalente à ingesta nutricional) é, atualmente, o marcador bioquímico mais utilizado para a avaliação da deficiência em iodo $(1,7)$; além de seu valor diagnóstico, é mais barata e tecnicamente mais simples que outros testes empregados para avaliar a deficiência de iodo, como dosagens séricas de TSH e tiroglobulina ou ultra-sonografia $(1,4,7)$. Além disso, estudos populacionais comparativos demonstram a correlação entre os valores obtidos por coleta de amostras de urina ao acaso (coleta casual) e as amostras de urina de 24 horas, em razão da distribuição gaussiana da concentração de iodo na urina $(9,10)$.

Apesar de as descrições de manifestações da carência de iodo serem precoces na história do Brasil (11-14), o primeiro inquérito nacional realizou-se apenas em 1955, sob coordenação da Divisão de Organização Sanitária do Ministério da Saúde; esse estudo, que compreendeu a avaliação de 86.217 escolares, detectou prevalência de bócio de $20,7 \%$ e delimitou as zonas de bócio endêmico. No período de 1974 a 1976, a SUCAM (Superintendência de Campanhas de Saúde Pública), do Ministério da Saúde, realizou o segundo inquérito nacional, envolvendo 421.752 escolares (203.251 meninos e 218.505 meninas), com idades entre 7 e 14 anos, mostrando prevalência de bócio de $14,1 \%$ (11,7\% para os meninos e 16,3\% para as meninas), ou seja, uma redução de apenas $6,5 \%$ no período de 20 anos. A partir de 1984, estabeleceramse, em vários estados, municípios-sentinela, visando a monitoração periódica dos níveis de controle da endemia. Um desses estudos, realizado em 1990 pelo INAN, Instituto Nacional de Alimentação e Nutrição, do Ministério da Saúde, demonstrou prevalência alarmante de bócio, indicando mau controle da endemia. Tal estudo mostrou a necessidade de atualização dos dados sobre a situação nacional da endemia de carência em iodo, inclusive associando o estudo de prevalência de bócio e a determinação da excreção urinária de iodo para a determinação da real dimensão do problema. Decidiu-se, então, no âmbito do Ministério da Saúde, a realização, em 1994, do $3^{\circ}$ Inquérito Nacional de Recenseamento da endemia de carência em iodo no Brasil, envolvendo a determinação de iodúria e a palpação da tiróide em escolares $(15,16)$.

Neste estudo, apresentamos o desenvolvimento e a validação de método semi-automatizado colorimétrico para a determinação de iodo na urina, ao mostrar os dados relativos à excreção urinária de iodo de 16.803 escolares de 401 municípios, objetivando mapear as áreas de persistência da carência de iodo no Brasil em 1994.

\section{MATERIAL E MÉTODOS}

\section{Grupo de estudo}

A partir dos dados obtidos pelo INAN, em 1990, sobre a prevalência de bócio nos municípios-sentinela de 5 estados 
brasileiros, o Ministério da Saúde concebeu um inquérito nacional para avaliação da endemia de carência em iodo, tendo sido o grupo de estudo composto por escolares de ambos os sexos, na faixa etária de 8 a 10 anos, usando como parâmetros a prevalência de bócio, a excreção urinária de iodo e a análise do conteúdo em iodo no sal consumido pelos escolares. Baseado no estudo de 1990, o inquérito adotou um sistema de amostragem híbrido, abrangendo os 13 municípios que haviam sido estudados (Palmeiras de Goiás, Cabeceiras, Flores de Goiás e Nova Roma em Goiás; Riachão e São Raimundo das Mangabeiras no Maranhão; Jequetinhonha e Mirabela em Minas Gerais; Cametá, Oeiras do Pará e Limoeiro do Ajurú no Pará; Peixe e Natividade em Tocantins), os municípios limítrofes a esses primeiros, e mais 15 municípios em cada um desses estados, escolhidos aleatoriamente, e 20 municípios dos demais estados brasileiros, escolhidos aleatoriamente, de forma a obter uma amostra representativa de cada unidade da federação (15-17).

Para o estudo dos níveis de iodo urinário, foram sorteadas pelo menos 20 crianças em cada município, sendo o número de crianças maior nas áreas endêmicas. No total, foram analisadas amostras de 16.803 crianças provenientes de 401 municípios (17).

\section{Coleta das amostras}

As amostras de urina foram colhidas por coleta casual, em potes plásticos de $50 \mathrm{~mL}$, identificadas (nome da criança, nome da escola, município e unidade da federação) e enviadas por via aérea (exceto São Paulo) para o Laboratório da Disciplina de Endocrinologia, Departamento de Medicina, da Escola Paulista de Medicina, Universidade Federal de São Paulo (UNIFESP), onde as amostras recebidas foram cadastradas por ordem de chegada, aliquotadas em tubos plásticos de $5 \mathrm{~mL}$ e armazenadas a $-20^{\circ} \mathrm{C}$ até a data da dosagem. Cada amostra foi identificada por um número de cadastro, nome da criança, idade e procedência (nome da escola, município, unidade da federação). Amostras de municípios do estado do Acre (Plácido de Castro, Sena Madureira e Cruzeiro do Sul) foram desprezadas devido a problemas ocorridos no envio das amostras (mistura das amostras de urina e de sal); devido a isso, os dados do município remanescente do Estado do Acre, Brasiléia, não são representativos da população daquele estado e foram analisados como município isolado.

Dosamos as amostras em ordem aleatória e de forma cega, sem que dispusséssemos de qualquer indicação quanto à procedência da amostra e ao exame palpatório da tiróide da criança estudada.

\section{Método de dosagem do iodo na urina}

Utilizamos o método recomendado pelo International Council for Control of Iodine Deficiency Disorders (ICCIDD), baseado na detecção indireta do iodo pela monitoração da redução do sulfato cérico amoniacal $(18,19)$ modificando-o pela utilização do persulfato de amônio como agente digestor das amostras de urina (20); além disso, desenvolvemos um método semi-automatizado em placas de
96 poços que, pelo prolongamento do tempo de reação de redução do cério, permite que a diferença de tempo de leitura dos poços não seja crítica.

Preparamos a curva-padrão a partir de uma solução padrão de iodo de $1 \mu \mathrm{g} / \mathrm{mL}$, obtida pela dissolução de 1,68 $\mathrm{mg}$ de iodato de potássio (equivalentes a $1 \mathrm{mg}$ de iodo) $\mathrm{em}$ 1 litro de água deionizada, armazenada a $4^{\circ} \mathrm{C}$ em alíquotas de $1 \mathrm{~mL}$. Para a realização dos diversos ensaios de iodúria, descongelamos as amostras dos pacientes a medir, agitamoas com vórtex para a re-suspensão do sedimento e pipetamos $250 \mu \mathrm{L}$ da curva-padrão $(0-200 \mu \mathrm{g} / \mathrm{L})$ e das amostras em tubos de ensaio de $13 \mathrm{~mm}$. Em seguida, adicionamos a cada tubo $1 \mathrm{~mL}$ de persulfato de amônio $1 \mathrm{M}$ com agitação e os aquecemos por $50-60$ minutos, a $90-95^{\circ} \mathrm{C}$, num bloco de aquecimento. A seguir, deixamos os tubos retornarem à temperatura ambiente e acrescentamos aos mesmos 3,5 mL de ácido arsenioso 0,2 $\mathrm{N}$ sob agitação. Após 15 minutos de repouso, adicionamos $350 \mu \mathrm{L}$ de sulfato cérico amoniacal sob agitação. Após 20 minutos, transferimos $200 \mu \mathrm{L}$ de cada tubo para a placa de 96 poços (MaxiSorp-NunC) e a incubamos a $37^{\circ} \mathrm{C}$ por cerca de 10 minutos. A seguir, lemos o resultado da reação no espectrofotômetro Novaspec II, Pharmacia LKB, na absorbância de $405 \mathrm{~nm}$, sendo o conteúdo de iodo nas amostras obtido por comparação das leituras com a curva-padrão e expresso em $\mu \mathrm{g} / \mathrm{L}$. Este método apresenta sensibilidade de $10 \mu \mathrm{g}$ de iodo/L, coeficiente de variação intra-ensaio de $12,6 \%$ para uma amostra de 89 $\mu \mathrm{g} / \mathrm{L}(\mathrm{n}=11)$ e de $2,3 \%$ para uma amostra de $277 \mu \mathrm{g} / \mathrm{L}$ (n $=11$ ), e coeficiente de variação inter-ensaio de $15,5 \%$ para uma amostra de $99 \mu \mathrm{g} / \mathrm{L}(\mathrm{n}=47)$ e de $10 \%$ para uma amostra de $262 \mu \mathrm{g} / \mathrm{L}(\mathrm{n}=47)$.

\section{Análise dos dados}

Os resultados foram agrupados pela procedência para o cálculo das medianas por município e unidade da federação. Para a análise de distribuição dos resultados, utilizamos os valores de corte da classificação do aporte de iodo que se correlaciona com a gravidade dos distúrbios associados à deficiência de iodo estabelecidos pelo ICCIDD, que determina que a mediana de iodúria $<25 \mu \mathrm{g} / \mathrm{L}$ representa deficiência grave, entre 25 e $49 \mu \mathrm{g} / \mathrm{L}$ deficiência moderada, entre 50 e $99 \mu \mathrm{g} / \mathrm{L}$ deficiência leve e acima de $100 \mu \mathrm{g} / \mathrm{L}$ suficiência de iodo (21). Além disso, subdividimos a faixa de valores maiores ou iguais a $100 \mu \mathrm{g} / \mathrm{L}$ em valores entre $100 \mathrm{e}<200$ $\mu \mathrm{g} / \mathrm{L}$ e outra de valores $\geq 200 \mu \mathrm{g} / \mathrm{L}$. Consideramos carentes todos os municípios com mediana inferior a $100 \mu \mathrm{g} / \mathrm{L}$ e aqueles onde, mesmo com mediana normal, mais de 10\% das amostras continha menos de $25 \mu \mathrm{g} / \mathrm{L}$ de iodo (21).

\section{RESULTADOS}

O estudo das 16.803 amostras de urina analisadas indica que a excreção urinária de iodo no Brasil, durante o ano de 1994, apresentava grande dispersão dos valores em relação à mediana, tendo sido encontradas tanto 
amostras com valores indetectáveis como maiores do que $200 \mu \mathrm{g} / \mathrm{L}$ em todas as unidades da federação. A distribuição das amostras pelos diversos Estados indica que só Tocantins (mediana do estado $=94 \mu \mathrm{g} / \mathrm{L}$ ) apresentava, globalmente, deficiência em iodo. Não foi possível analisar o Acre porque a amostra não foi representativa de sua população.

Quando analisamos os resultados individuais de cada município, observamos grande heterogeneidade na distribuição das amostras dentro das faixas estabelecidas. Observamos medianas indicativas de deficiência em iodo em 85 municípios. Em 4, os níveis foram compatíveis com deficiência moderada de iodo (valores

Tabela 1. Municípios da região norte do Brasil com deficiência de iodo grave (medianas de iodúria abaixo de 25 $\mu \mathrm{g} / \mathrm{L}$ ), moderada (medianas de iodúria entre 25 e $49 \mu \mathrm{g} / \mathrm{L}$ ) e leve (medianas de iodúria entre 50 e $99 \mu \mathrm{g} / \mathrm{L}$ ) em 1994-1996. Os municípios destacados apresentam os maiores graus de deficiência.

\begin{tabular}{|c|c|c|}
\hline ESTADO & Município & Mediana $(\mu \mathrm{g} / \mathrm{L})$ \\
\hline Acre & Brasiléia & $64(n=36)$ \\
\hline Amapá & $\begin{array}{l}\text { Calcoene } \\
\text { Macapá }\end{array}$ & $\begin{array}{l}70(n=63) \\
85(n=141)\end{array}$ \\
\hline Amazonas & $\begin{array}{c}\text { Careiro da Várzea } \\
\text { Coari } \\
\text { Guajará } \\
\text { Humaitá } \\
\text { Lábrea } \\
\text { Tefé }\end{array}$ & $\begin{array}{l}83(n=40) \\
89(n=40) \\
92(n=38) \\
82(n=37) \\
89(n=40) \\
77(n=40)\end{array}$ \\
\hline Pará & $\begin{array}{c}\text { Bagre } \\
\text { Baião } \\
\text { Curionópolis } \\
\text { Curralinho } \\
\text { Limoeiro do Ajuru } \\
\text { Marabá } \\
\text { Oeiras do Pará } \\
\text { Portel } \\
\text { Santarém } \\
\text { Viseu }\end{array}$ & $\begin{array}{l}59(n=40) \\
74(n=27) \\
91(n=40) \\
65(n=40) \\
74(n=39) \\
78(n=40) \\
83(n=40) \\
80(n=39) \\
89(n=40) \\
79(n=38)\end{array}$ \\
\hline Rondônia & Jaru & $81(n=36)$ \\
\hline Roraima & Bonfim & $79(n=11)$ \\
\hline Tocantins & $\begin{array}{c}\text { Almas } \\
\text { Ananás } \\
\text { Aparecida do Rio Negro } \\
\text { Araguacema } \\
\text { Arraias } \\
\text { Augustinópolis } \\
\text { Figueirópolis } \\
\text { Gurupi } \\
\text { Itacajá } \\
\text { Monte do Carmo } \\
\text { Natividade } \\
\text { Palmas } \\
\text { Palmeirópolis } \\
\text { Paraíso do Tocantins } \\
\text { Paraná } \\
\text { Peixe } \\
\text { São Valério da Natividade } \\
\text { Silvanópolis } \\
\text { Tocantínia } \\
\text { Wanderlândia } \\
\text { Xambioá }\end{array}$ & $\begin{array}{l}33(n=36) \\
88(n=37) \\
77(n=28) \\
56(n=59) \\
34(n=45) \\
87(n=29) \\
73(n=33) \\
84(n=47) \\
69(n=32) \\
64(n=40) \\
91(n=32) \\
96(n=35) \\
74(n=40) \\
92(n=52) \\
26(n=39) \\
67(n=36) \\
62(n=37) \\
92(n=31) \\
77(n=29) \\
82(n=19) \\
95(n=36)\end{array}$ \\
\hline
\end{tabular}

entre 25 e $<50 \mu \mathrm{g} / \mathrm{L})$ : Cocos, na Bahia, com mediana de $44 \mu \mathrm{g} / \mathrm{L}$ e Almas, Arraias e Paraná, em Tocantins, com medianas de 33, 34 e $26 \mu \mathrm{g} / \mathrm{L}$, respectivamente. Nos outros 81 municípios, os níveis indicam uma deficiência leve (valores $\geq 50 \mathrm{e}<100 \mu \mathrm{g} / \mathrm{L}$ ) (tabelas 1 a 4 ). A localização desses 85 municípios indica, no mapa do Brasil, uma concentração nas bacias Amazônica, do Meio-Norte, Paranaica e Sanfranciscana (figura 1).

Apesar de apresentarem valores medianos iguais ou maiores a $100 \mu \mathrm{g} / \mathrm{L}, 35$ outros municípios evidenciaram que mais de $10 \%$ das amostras continham níveis de iodo inferiores a $25 \mu \mathrm{g} / \mathrm{L}$, o que indica, também, ingestão insuficiente de iodo em uma parcela significativa da população. A tabela 5, que apresenta a distribuição das amostras por escola em Viana, Espírito Santo, exemplifica bem esse problema: mesmo com $27,6 \%$ das crianças apresentando valores $<25 \mu \mathrm{g} / \mathrm{L}$, a mediana dos valores foi $126 \mu \mathrm{g} / \mathrm{L}$, devido às diferenças entre escolas; enquanto nas escolas Aracatiba e Hercília J. Azevedo predominam os valores baixos (5/8 e 6/7), na Castorino R. Siqueira todas as 6 amostras apresentaram teores normais de iodo.

\section{DISCUSSÃo}

Tradicionalmente, os estudos de monitoração da deficiência endêmica de iodo baseavam-se, exclusivamente, no estudo da prevalência de bócio e subesti-

Tabela 2. Municípios da região nordeste do Brasil com deficiência de iodo grave (medianas de iodúria abaixo de 25 $\mu \mathrm{g} / \mathrm{L}$ ), moderada (medianas de iodúria entre 25 e $49 \mu \mathrm{g} / \mathrm{L}$ ) e leve (medianas de iodúria entre 50 e $99 \mu \mathrm{g} / \mathrm{L}$ ) em 1994-1996. O município destacado apresenta o maior grau de deficiência.

\begin{tabular}{lcc} 
ESTADO & Município & $\begin{array}{c}\text { Mediana } \\
(\mu \mathrm{g} / \mathrm{L})\end{array}$ \\
Alagoas & Atalaia & $91(n=30)$ \\
& Coruripe & $72(n=31)$ \\
Bahia & Cão de Açúcar & $98(n=33)$ \\
& Cocópos & $55(n=27)$ \\
Maranhão & Correntina & $64(n=34)$ \\
& Alto Parnaíba & $68(n=27)$ \\
Paraíba & Zé Doca & $88(n=24)$ \\
& Arara & $92(n=22)$ \\
Pernambuco & Juazeirinho & $90(n=40)$ \\
Piauí & Vitória de Santo Antão & $89(n=38)$ \\
& Conceição do Canindé & $79(n=36)$ \\
Rio Grande do Norte & Oeiras & $66(n=51)$ \\
& Santo Inácio & $61(n=37)$ \\
& Augusto Severo & $67(n=41)$ \\
& Ceará-mirim & $97(n=40)$ \\
& Nova Cruz & $97(n=23)$ \\
& Serra de São Bento & $94(n=40)$ \\
\hline
\end{tabular}


Tabela 3. Municípios da região centro-oeste do Brasil com deficiência de iodo moderada (medianas de iodúria entre 25 e $49 \mu \mathrm{g} / \mathrm{L}$ ) e leve (medianas de iodúria entre 50 e 99 $\mu \mathrm{g} / \mathrm{L})$ em 1994-1996.

\begin{tabular}{lcc}
\hline ESTADO & Município & $\begin{array}{c}\text { Mediana } \\
(\mu \mathrm{g} / \mathrm{L})\end{array}$ \\
Goiás & Alvorada do Norte & $83(\mathrm{n}=27)$ \\
& Cabeceiras & $95(\mathrm{n}=63)$ \\
Edéia & $86(n=22)$ \\
Guarani de Goiás & $70(n=34)$ \\
Monte Alegre de Goiás & $61(n=34)$ \\
& Nova Roma & $90(n=30)$ \\
Planaltina & $96(n=24)$ \\
Mato Grosso do Sul & $66(n=32)$ \\
& São Domingos João d'Aliança & $74(n=28)$ \\
& Angélica & $85(n=40)$ \\
& Paranhos & $94(n=30)$ \\
\hline
\end{tabular}

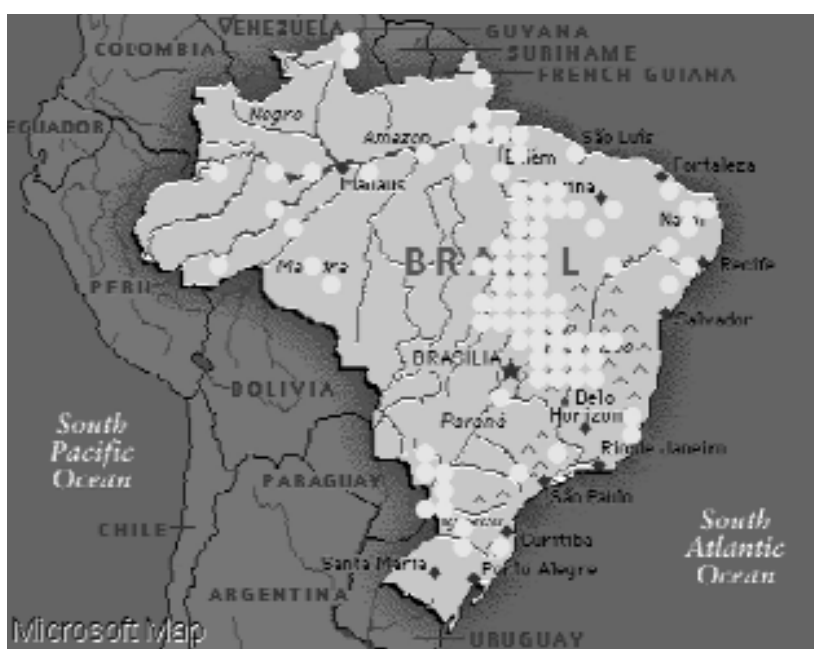

Figura 1. Localização dos municípios (em branco) com deficiência leve e moderada de iodo no Brasil.

mavam a real deficiência de iodo, pois deficiências leves de iodo, suficientes para que existam adaptações funcionais, como aumento da captação tiroidiana, da depuração renal de iodo e da sensibilidade ao TSH, podem não se manifestar como bócio (22); além disso, a inspeção e palpação da tiróide, usada na maior parte dos inquéritos para classificação dos bócios, discrimina mal a tiróide normal e os bócios pequenos, tendo sensibilidade inferior à ultrassonografia $(23,24)$.

Devido a isso, recomenda-se atualmente que a avaliação do volume tiroidiano (de preferência por ultra-sonografia) seja associada a outro parâmetro, sendo a determinação da excreção urinária de iodo de escolares, em conjunto com a avaliação do volume tiroidiano, a forma mais prática de monitorar o controle da deficiência endêmica de iodo (4).

Este estudo fez parte do terceiro inquérito sobre o controle da endemia no Brasil e foi a primeira
Tabela 4. Municípios das regiões sudeste e sul do Brasil com deficiência de iodo moderada (medianas de iodúria entre 25 e $49 \mu \mathrm{g} / \mathrm{L}$ ) e leve (medianas de iodúria entre 50 e 99 $\mu \mathrm{g} / \mathrm{L})$ em 1994-1996.

\begin{tabular}{lcc}
\hline ESTADO & Município & Mediana $(\boldsymbol{\mu g} / \mathbf{L})$ \\
Espírito Santo & Linhares & $88(n=24)$ \\
Minas Gerais & Brasilia de Minas & $62(n=31)$ \\
& Coração de Jesus & $81(n=40)$ \\
& Itajubá & $75(n=20)$ \\
& Januária & $92(n=38)$ \\
& Mirabela & $89(n=37)$ \\
São Paulo & Montes Claros & $76(n=36)$ \\
Paraná & São João da Ponte & $92(n=67)$ \\
& Sarapuí & $83(n=22)$ \\
& Barracão & $92(n=19)$ \\
Santa Catarina & Cascavel & $92(n=35)$ \\
& Mamboré & $86(n=39)$ \\
& Missal & $64(n=28)$ \\
\hline
\end{tabular}

Tabela 5. Amostras de iodúria em diversas escolas do município de Viana, Espírito Santo.

\begin{tabular}{lc}
\hline Município: Viana (ES) & lodúria $(\boldsymbol{\mu g} / \mathbf{L})$ \\
\hline Escola Aracatiba & $<25$ \\
& $<25$ \\
& $<25$ \\
& 343 \\
& $<25$ \\
& $<25$ \\
Escola Castorino R. Siqueira & 142 \\
& 126 \\
& 154 \\
& 159 \\
Escola Dr. Donizete Santos & 332 \\
& 324 \\
& 257 \\
& 135 \\
& 240 \\
& $<25$ \\
& 35 \\
& 163 \\
& 197 \\
& 81 \\
& 244 \\
& 126 \\
& $<25$ \\
& 37 \\
& 87 \\
& 149 \\
& 73 \\
& 25 \\
& 29 \\
& \\
& \\
&
\end{tabular}

determinação de iodo urinário em escala nacional. Como os dois inquéritos anteriores basearam-se na prevalência de bócio para avaliar a endemia, era previsível que, associando a determinação do iodo urinário aos dados de prevalência de bócio, o presente estudo revelasse não somente as áreas tradicionais de bócio endêmico como também regiões de aporte limítrofe ou levemente deficiente de iodo. 
Dos 401 municípios estudados, observamos deficiência em iodo em 85 municípios, sendo de grau moderado ( $\geq 25$ e $<50 \mu \mathrm{g} / \mathrm{L})$ em Cocos, na Bahia, e Almas, Arraias e Paraná, no Tocantins, e nos demais de grau leve (valores $\geq 50 \mathrm{e}<100 \mu \mathrm{g} / \mathrm{L}$ ). Em outros 35 municípios, a mediana dos valores foi normal, mas parcela significativa da população (mais de $10 \%$ das crianças) apresentou níveis de iodo inferiores a $25 \mu \mathrm{g} / \mathrm{L}$; isto se deveu à presença de amostras com conteúdos muito heterogêneos de iodo no mesmo município, fato também posteriormente observado em Ouro Preto, Minas Gerais (25).

Alguns fatores podem explicar essa variação: a localização rural ou urbana da escola, as grandes dimensões de alguns municípios (principalmente no Norte e Centro-Oeste), o isolamento de localidades e distritos da sede do município, a inclusão de escolas públicas e privadas na amostra e o horário das coletas (tendo em vista que a concentração urinária de iodo varia durante o dia). Um outro fator a ser considerado é que existindo um programa de suplementação alimentar de iodo, ocorre uma dispersão muito maior dos resultados relacionada ao grau de conscientização e ao poder aquisitivo da população.

A existência de regiões carentes em iodo é consequência do ciclo do elemento na natureza: o iodo presente nos oceanos em percentagens bastante regulares (em torno de $50 \mu \mathrm{g} / \mathrm{L}$ ) evapora e é concentrado nas nuvens que são levadas para os continentes; as chuvas exercem um duplo papel, de um lado enriquecendo as camadas superficiais do solo com iodo e de outro contribuíndo para a "lavagem" e expoliação do iodo geologicamente presente no solo, que é transportado pelos rios de volta para o oceano (26). Desta forma, em áreas montanhosas de formação recente, como Himalaia, Andes e Alpes, onde o solo foi expoliado pela ação das chuvas e glaciações, e em áreas sedimentares das grandes bacias hidrográficas, como a do Ganges e do Mekong, sujeitas a enchentes freqüentes, a pobreza geológica de iodo faz com que ele seja insuficiente em toda a cadeia alimentar.

No Brasil, observamos que até 1994 as áreas de deficiência em iodo correspondem a regiões de bacias sedimentares, compostas de estrutura geológica recente, abrangendo as bacias Sanfranciscana, do Meio-norte, Amazônica e Paranaica. Em outras áreas, atribuímos a elevada prevalência de deficiência em iodo a fatores sócio-econômicos, como colonização e urbanização de áreas da Região Norte. A derrubada da floresta com substituição por plantações e pastagens e a aquisição de hábitos urbanos contribuem para que se rompa o equilíbrio natural e têm como conseqüência uma rápida e progressiva expoliação do iodo do solo (27-29).
Os resultados deste estudo, em associação com as informações relativas à palpação da tiróide nas crianças estudadas, que indicou ausência de bócio nos escolares (16), demonstram que, em 1994, as manifestações extremas da carência de iodo, como o cretinismo e os bócios muito volumosos, eram esporádicas no Brasil e estavam de acordo com o levantamento feito em 1975, que encontrou apenas um caso de bócio visível entre 421.752 escolares estudados (30). No entanto, o achado de deficiência de grau leve a moderado em uma porcentagem significativa dos municípios estudados $(29,9 \%)$ sugeriu que as ações de combate à deficiência de iodo no Brasil não era completas e devem ter como meta a profilaxia e tratamento das manifestações sutis e subvalorizadas da baixa ingesta de iodo.

De fato, o conceito de distúrbios associados à deficiência de iodo é muito mais abrangente, englobando não só as manifestações clássicas de bócio e cretinismo endêmicos, como também todas as conseqüências clínicas e sócio-econômicas do aporte nutricional deficiente (1-7); as fases da vida onde essas manifestações são mais graves são, principalmente, os períodos intrauterino e neonatal (devido ao papel fundamental dos hormônios tiroidianos no desenvolvimento neurológico) e a gestação (1-7). Além disso, a incidência global das doenças tiroidianas é significativamente maior em populações carentes em iodo, mesmo em grau leve, que em áreas suficientes (4); em particular, bócio, autonomia tiroidana, hipertiroidismo induzido por iodo, hipotiroidismo congênito transitório e definitivo são mais comuns em regiões deficientes $(4,31,32)$. O padrão de incidência de neoplasias malignas da tiróide também é relacionado à ingesta de iodo: em áreas carentes, há um aumento da incidência de carcinomas foliculares e anaplásicos, enquanto em populações suficientes incidem proporcionalmente mais carcinomas papilíferos $(33,34)$.

Entretanto, muito mais importantemente que o aumento da morbidade das tiropatias, a deficiência em iodo tem como conseqüência lesão cerebral e manifestações neurológicas (alterações psicomotoras, redução da capacidade de concentração e aprendizado), hipoevolutismo físico e problemas materno-infantis (redução da fertilidade, aumento da incidência de malformações congênitas, abortos, prematuridade, mortalidade perinatal e infantil) (1-7).

Desta forma, além da elevação das despesas com o atendimento de saúde das populações carentes, a deficiência em iodo tem outras importantes conseqüências sócio-econômicas: desproporção entre os investimentos em educação e o retorno (altas taxas de 
repetência e evasão escolares), baixa capacidade produtiva no trabalho e produção animal reduzida (baixa fertilidade das matrizes, engorda insuficiente, baixa produção de ovos, leite e lã) (1-7). A associação desses fatores contribui para que as populações mesmo levemente deficientes apresentem um desenvolvimento econômico e qualidade de vida inferiores às que têm um aporte satisfatório de iodo. Coincidentemente, os focos endêmicos caracterizados neste trabalho localizavam-se em sua quase totalidade em regiões menos desenvolvidas do país.

Possivelmente, a deficiência em iodo associada à desnutrição protéico-calórica altamente prevalente faz com que se perpetue o ciclo de pobreza que impede que essas populações se desenvolvam. Certamente, o retorno econômico devido à redução dos gastos com saúde e educação e à melhoria dos parâmetros econômicos que decorreriam da extinção da deficiência em iodo são incomparavelmente maiores que o investimento de cerca de US\$ 0,05 por habitante por ano necessários para manter o programa de iodação do sal.

Os resultados deste estudo mostravam que, a despeito das medidas de combate à deficiência em iodo, em 1994 ainda persistiam bolsões de carência endêmica de iodo no Brasil, alguns dos quais em regiões que eram consideradas livres do problema. Como predominam áreas de carência leve a moderada, as manifestações carenciais extremas, como bócios volumosos e cretinismo, estavam sob controle, mas devemos concentrar os esforços no combate às manifestações sutis, porém quantitativamente muito mais importantes da endemia.

Isso levou a uma série de ações do Ministério da Saúde (3) que causaram a elevação do consumo do iodo, o que observamos por uma série de dados disponíveis. Assim, em 2000 realizou-se outro levantamento em 21 municípios das regiões norte, centrooeste e sudeste, sob o patrocínio da ICCIDD $(3,10,33)$, nos municípios onde o inquérito revelado neste trabalho havia evidenciado deficiência leve e moderada. Apesar do número menor de amostras $(\mathrm{n}=$ 1.013), observou-se, surpreendentemente, elevação da excreção urinária de iodo em relação a 1994, sendo que $86,5 \%$ dos escolares apresentava iodúria acima de $300 \mu \mathrm{g} / \mathrm{L}$, dos quais metade acima de $500 \mu \mathrm{g} / \mathrm{L}$ (3). Além disso, a ultra-sonografia realizada em 2.013 crianças observou a tiróide aumentada em apenas 28 delas $(1,4 \%)$ (33). Levantamentos regionais e de escopo menor também têm confirmado a suficiência de aporte iódico em populações brasileiras $(34,35)$.

É claro que o excesso de iodo também pode causar uma série de doenças da tiróide (3) e deve-se estar atento permanentemente para a suplementação adequada de iodo no sal. O método de dosagem do iodo urinário desenvolvido neste trabalho apresenta as características de simplicidade e eficiência necessários para este tipo de estudo populacional.

Baseados no presente estudo, que indicava deficiência na ingestão de iodo em 1994 e de novas evidências provenientes de estudos limitados (10,3335), mas indicativos de excesso na ingestão de iodo, recomendamos que o Ministério da Saúde retome suas avaliações periódicas sobre o estado nutricional de iodo e cumpra a legislação vigente que trata da iodação do sal no Brasil, identificando os fatores que prejudicam sua efetividade, obedecendo, inclusive, às determinações da OMS; além disso, consideramos fundamental que os profissionais da saúde e dos órgãos governamentais sejam conscientizados permanentemente sobre o conceito de distúrbios associados à carência e ao excesso de iodo.

\section{AGRADECIMENTOS}

Este trabalho foi financiado pela CAPES (Fundação Coordenadoria de Aperfeiçoamento de Pessoal de Ensino Superior), na forma de bolsa de doutorado a RZE, e pela UNICEF (Fundo das Nações Unidas para a Infância), por meio de auxílio ao Ministério da Saúde. Agradecemos o empenho técnico de Yvone F. Bianco e Ângela Faria.

\section{REFERÊNCIAS}

1. lodine status worldwide: World health Organization (WHO) Global Database on lodine Deficiency. ISBN 924159200. Available at: <http://www.who.org $>$.

2. Hetzel BS. Eliminating iodine deficiency disorders-the role of the International Council in the global partnership. Bull WHO 2002;80:410-7.

3. Knobel M, Medeiros-Neto G. Moléstias associadas à carência crônica de iodo. Arq Bras Endocrinol Metab 2004;48:5361.

4. Dunn JT. Seven deadly sins in confronting endemic iodine deficiency and how to avoid them. J Clin Endocrinol Metab 1996;81:1332-5.

5. Delange $F$. The disorders induced by iodine deficiency. Thyroid 1994;4:107-28.

6. Boyages SC. lodine deficiency disorders. J Clin Endocrinol Metab 1993;77:587-91.

7. International Council for Control of lodine Deficiency Disorders. Available at: <http://www.iccidd.org>.

8. Gaitan E, Dunn JT. Epidemiology of iodine deficiency. Trends Endocrinol Metab 1992;3:170-5.

9. Bourdoux $P$, Thilly $C$, Delange $F$, Ermans AM. A new look at old concepts. In: Dunn JT, Pretell EA, Daza CH, Viteri FE (eds). Towards the eradication of endemic goiter, cretinism, and iodine deficiency. Washington: PAHO, 1986. pp. 11529. 
10. Pretell EA, Delange F, Hostalek U, Corigliano S, Barreda L, Higa AM, et al. lodine nutrition improves in Latin America. Thyroid 2004;590-9.

11. Medeiros-Neto G. História do bócio endêmico no Brasil. Origens e causas. São Paulo: Secretaria de Cultura, Esportes e Turismo, 1975.

12. Medeiros-Neto G. Cento e oitenta anos de bócio endêmico no Brasil. Arq Bras Endocrinol Metab 1982;26:120-8.

13. Dantas LM. Endemic goiter in Brazil. In: Dunn JT, Pretell EA Daza $\mathrm{CH}$, Viteri FE (eds). Towards the eradication of endemic goiter, cretinism, and iodine deficiency. Washington: PAHO, 1986. pp. 271-5.

14. Silva YSP. Programa Nacional de Controle dos Distúrbios por Deficiência de lodo, Ministério da Saúde. Disponível em: $<$ http://www.lats.org $>$.

15. Instituto Nacional de Alimentação e Nutrição, Fundação Nacional de Saúde. Manual de combate aos distúrbios por deficiência de iodo. Ministério da Saúde, 1996.

16. Ministério da Saúde. $3^{\circ}$ Inquérito Nacional para avaliação da endemia de carência em iodo. Brasília, 1994

17. Barros $F$, Victora $C$. Inquérito para avaliação da prevalência de bócio em escolares brasileiros. Epicentro: Universidade Federal de Pelotas, 1994

18. Sandell ED, Kolthoff IM. Microdetermination of iodine by a catalytic method. Mikrochemica Acta 1937;1:9-25.

19. Dunn JT, Crutchfield HE, Gutekunst R, Dunn AD. Two simple methods for measuring iodine in urine. Thyroid 1993;3:11923.

20. Pino S, Fang S-L, Braverman LE. Ammonium persulfate: a safe alternative oxidizing reagent for measuring urinary iodine. Clin Chem 1996;42:239-43.

21. Dunn JT, Crutchfield HE, Gutekunst R, Dunn AD. Methods for measuring iodine in urine. Wageningen: ICCIDD, 1993.

22. Dumont JE, Ermans AM, Maenhaut C, Coppee F, Stanbury JB. Large goitre as a maladaptation to iodine deficiency. Clin Endocrinol 1995;43:1-10.

23. Berghout A, Wiersinga WM, Smits NJ, Touber JL. The value of thyroid volume measured by ultrasonography in the diagnosis of goitre. Clin Endocrinol 1988;28:409-14.

24. Zimmermann MB, Molinari L, Spehl M, Weidinger-Toth J, Podoba J, Hess S, et al. Towards a consensus on reference values for thyroid volume in iodine replete schoolchildren: results of a workshop on interobserver and interequipment variation in sonographic measurement of thyroid volume. Eur J Endocrinol 2001;144:213-20.

25. Nimer M, Silva ME, Dutra de Oliveira JE. Associações entre iodo no sal e iodúria em escolares, Ouro Preto, MG. Rev Saúde Pública 2002;36:500-4.
26. Koutras DA. lodine: distribution, availability, and effects of deficiency on the thyroid. In: Dunn JT, Pretell EA, Daza CH, Viteri FE (eds). Towards the eradication of endemic goiter, cretinism, and iodine deficiency. Washington: PAHO, 1986. pp. 15-27.

27. Vieira-Filho JPB. Considerações a propósito da inexistência de bócio entre os indígenas brasileiros Xikrin. Rev Ass Med Bras 1972; 18:345-8.

28. Vieira-Filho JPB. O bócio entre os índios brasileiros. Rev Ass Med Bras 1981;27:285-7.

29. Vieira-Filho JPB, Maciel RMB, Juliano Y. Níveis séricos da tiroglobulina dos silvícolas Xicrin e Paracanã. Arq Bras Endocrinol Metab 1984;28:57-60.

30. Medeiros-Filho A. Bócio endêmico: levantamento de sua prevalência em todo o território brasileiro por micro-regiões homogêas. Rev Bras Malariolog Doenças Trop 1976;28:1-4

31. Hall $\mathrm{R}$, Lazarus $\mathrm{JH}$. Changing iodine intake and the effect on thyroid disease. Br Med J 1987;294:721-2.

32. Lima N, Medeiros-neto G. Transient thyrotoxicosis in endemic goitre patients following exposure to a normal iodine intake. Clin Endocrinol 1984;21:631-7.

33. Rossi AC, Tomimori E, Camargo R, Medeiros-Neto G. Searching for iodine deficiency disorders in schoolchildren from Brazil: the Thyromobil project. Thyroid 2001;11:661-3.

34. Duarte CG, Tomimori EK, Boriolli RA, Ferreira JE, Catarino RM, Camargo RY, et al. Echographic evaluation of the thyroid gland and urinary iodine concentration in schoolchildren from various regions of the State of São Paulo, Brazil. Arq Bras Endocrinol Metab 2004;48:842-8.

35. Saab ARM. Excreção urinária de iodo e prevalência de bócio em escolares de Campo Grande, MS. [tese de mestrado] Universidade Federal de Mato Grosso do Sul, 2000.

\section{Endereço para correspondência:}

Rui M.B. Maciel

Laboratório de Endocrinologia Molecular

Disciplina de Endocrinologia, Departamento de Medicina

Escola Paulista de Medicina, Universidade

Federal de São Paulo

Rua Pedro de Toledo 781, $12^{\circ}$ andar

04032-029 São Paulo, SP

Fax: (1 1) 5084-5231

E-mail: rmbmaciel-endo@pesquisa.epm.br 